Este artículo presenta las respuestas que los gobiernos locales de Iztapalapa, Morelia, Xalapa y Cuernavaca, han tenido frente a la contingencia de la pandemia por CovID-19. Las respuestas se recopilaron de los alcaldes, de un cuestionario en línea aplicado a ciudadanos de Cuernavaca, y de las acciones publicadas por la alcaldía. Las acciones tomadas, fueron medidas urgentes para cumplir las consignas emitidas por la autoridad sanitaria nacional de "quedarse en casa" y "guardar una sana distancia", ante la pandemia; pero también, ante las consecuencias de estas medidas: no poder ir a trabajar, proveer alimentos y carecer de condiciones de vivienda adecuadas.

Palabras clave: respuestas, gobiernos locales, pandemia covID-19

\title{
Respuestasde los gobiernos locales a la pandemia por COVID-19
}

Medardo Tapia Uribe

medardo@unam.mx medardotapia@gmail.com

\section{Introducción}

Ciudadanos y autoridades de gobiernos municipales, consideran que los gobiernos locales tienen la obligación de ofrecer una respuesta frente a la contingencia de salud pública que enfrentan en sus municipios, como en el resto de México y el mundo; pues son la instancia de gobierno más cercana a los ciudadanos y deberían de ser quienes enfrentaran los problemas de salud ocasionados por el Covid-19 y las consecuencias por cumplir con la consigna de "Quédate en casa", decretada por la autoridad sanitaria nacional y los gobiernos estatales - la segunda autoridad sanitaria constitucional-.

Dentro de sus facultades constitucionales, los gobiernos locales municipales de México, han tomado acciones para contribuir a cumplir las medidas que ha dictado la autoridad sanitaria nacional; pero también para colaborar y apoyar a las familias y ciudadanos

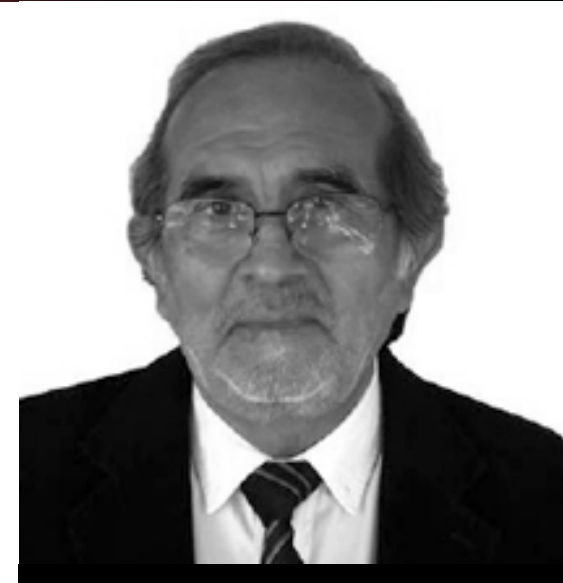

Investigador definitivo de tiempo completo del CRIM adscrito al programa Estudios en Gobierno y Políticas Públicas 
ca

¿Quiénes son los que se contagian?

La población que sale a trabajar

todas las mañanas." para prevenir la enfermedad, así como las consecuencias de quedarse en casa y no poder ir a trabajar, especialmente para quienes "viven al día", no son asalariados y no tienen seguridad social.

Esta colaboración tiene como propósito presentar las acciones — de las autoridades locales y los municipios seleccionados-, para enfrentar la pandemia por covid-19 y las consecuencias de cumplir con la consigna de "Quédate en casa", bajo las condiciones de habitabilidad de la vivienda y las actividades económicas que los habitantes, de dichos lugares, desarrollan para poder vivir. Se seleccionaron las ciudades y gobiernos locales de: Iztapalapa, Morelia, Xalapa y Cuernavaca. Cabe aclarar que dichos lugares no son representativos de gobiernos locales y ciudades del país; son solo casos de estudio, cuyos datos fueron recopilados por entrevistas a ciudadanos y a sus autoridades locales, por medio de las tecnologías de la comunicación —redes sociales- y cuestionarios en línea, elaborados colectivamente por diversos investigadores de la unAm y otras universidades del país, mediante una estrategia llamada "bola de nieve". Las entrevistas abiertas a los alcaldes, fueron convocadas por la Red de Investigadores en Gobiernos Locales Mexicanos (IGLOM).

\section{Iztapalapa}

Iztapalapa ha sido la alcaldía del país con el mayor número de contagios en el país; al 8 de junio encabezaba la lista con 5819 casos positivos acumulados y casi 700 defunciones. Aunque, en la última semana tuvo una disminución de 7.44 por cada 100 mil habitantes,

Tabla 1

Casos positivos acumulados y activos al 8 de junio

\begin{tabular}{|l|c|c|c|c|c|}
\hline \multicolumn{1}{|c|}{ Municipio } & $\begin{array}{c}\text { Casos } \\
\text { Positivos } \\
\text { Acumulados } \\
\text { (cPA) }\end{array}$ & $\begin{array}{c}\text { Casos } \\
\text { Positivos } \\
\text { activos }\end{array}$ & $\begin{array}{c}\text { Tasa Casos } \\
\text { Positivos } \\
\text { Acumulados } \\
\text { (pccmh) }\end{array}$ & $\begin{array}{c}\text { Tasa Casos } \\
\text { Positivos } \\
\text { Activos } \\
\text { (pccmh) }\end{array}$ & Población \\
\hline Cuernavaca & 419 & 52 & 104.9 & 13.0 & 399,426 \\
\hline $\begin{array}{l}\text { Zona Metropolitana } \\
\text { de Cuernavaca }\end{array}$ & 736 & 100 & 73.3 & 10.0 & $1,003,968$ \\
\hline Iztapalapa & 5,819 & 714 & 320.5 & 39.3 & $1,815,551$ \\
\hline Morelia & 255 & 59 & 30.9 & 7.1 & 825,585 \\
\hline Xalapa & 107 & 28 & 20.8 & 5.4 & 513,443 \\
\hline Nacional & & & & 14.4 & \\
\hline
\end{tabular}

Fuente: Elaboración propia, datos obtenidos de Centro de Investigaciones en Geografía Ambiental (2020)

"pccmh: personas por cien mil habitantes 
en comparación de la semana anterior. Iztapalapa tiene una población estimada de 1815 551 habitantes, para 2020 y, según su alcaldesa, es la cuarta alcaldía más poblada del país.

La alcaldesa de Iztapalapa Clara Marina Brugada Molina, señala el porqué de esta situación y algunas de las acciones que han tomado:

¿Quiénes son los que se contagian? La población, la que sale a trabajar todas las mañanas. Las gentes que salen a trabajar (...) que utiliza el metro (...) la que toma los microbuses, aunque haya bajado la movilidad (...) llena los microbuses (...) el metro (...) donde no hay distanciamiento social (...) son el principal problema de contagio de esta población. Esta gente no puede dejar de salir a trabajar (...) porque tiene que sobrevivir (...) de otra manera estaríamos enfrentado estallidos sociales... (Entrevista con alcaldesa de Iztapalapa, 2020).

La alcaldesa señala que, según la Encuesta Origen Destino de 2017, en Iztapalapa un millón setecientos veinticuatro mil personas, se mueven en transporte público, un millón en vehículos particulares, cuarenta y ocho mil en bicicleta y noventa mil personas caminando. $\mathrm{Y}$ es que aquí, señala, la población vive al día; porque en Iztapalapa se concentra la pobreza de la ciudad y 600 mil habitantes, que solo tienen agua cada diez días. Así enfrentaban la pandemia y la gente no puede obedecer el aislamiento social.

La alcaldía tuvo que atender, en primer lugar, la falta de agua de las familias, por lo cual se distribuyen mil viajes de pipas al día, desde el 18 de marzo; esto les "destrozó" el presupuesto. Otras de las acciones que tomaron, fue la desinfección de los espacios públicos de 670 unidades habitacionales, el cierre de 550 espacios públicos y la aplicación de un programa al que denominaron "Mercomuna", que es un programa en el que se otorgan 300 pesos en vales quincenales, a 116000 beneficiarios del padrón de Liconsa, para canjearlos en los comercios y mercados locales. Así, se apoya a los sectores más pobres de Iztapalapa y a los micronegocios locales; también implementaron un programa al que llamaron "Canasta verde de diez por diez", de alimentos perecederos, traídos directamente del campo a la ciudad.

En Iztapalapa hay 354 tianguis, en donde 546000 personas compran o venden y 350 brigadas municipales intervienen para que las personas entren con cubrebocas. La alcaldía negoció con los tianguistas para que no se instalara ningún tianguis y prevenir que 500000 personas fueran potencialmente factor de contagio. A cambio de esto, el gobierno federal y el gobierno de la Ciudad de México, otorgaron créditos de 25 mil pesos para las personas del comercio no formal, con cuatro meses de gracia, para que después, la gente vaya pagando como pueda. Con estas acciones se ha logrado detener la movilidad en Iz-

$\boldsymbol{c c}$ tapalapa, para que no se tengan tantos con...no puede dejar de salir a trabajar [...] porque tiene que sobrevivir.. Esto significa que vive al día." tagios. Para el 8 de junio, Iztapalapa había reducido a 7.44 casos positivos por cada 100 mil habitantes, un descenso de 135 casos totales entre semanas consecutivas (Centro de Investigaciones en Geografía Ambiental, 2020). Sin embargo, Iztapalapa presenta 714 positivos activos y una tasa de 39.3 personas por 100 mil habitantes (al 8 de junio), casi el triple de la nacional. 


\section{Morelia}

Morelia fue otro de los gobiernos municipales investigados, con la finalidad de conocer las medidas que han tomado para enfrentar la pandemia y cumplir con los lineamientos sanitarios de quedarse en casa y mantener una distancia social.

El municipio de Morelia, capital del estado de Michoacán, tiene una población estimada de 825585 habitantes (para 2020). Morelia, con un poco más de la mitad de la población de Iztapalapa, presentó una tasa de casos positivos activos de 7.1 personas por cada 100 mil habitantes, al 8 de junio; cinco veces menor que Iztapalapa. Según el alcalde, ha tenido buenos resultados para enfrentar la pandemia porque, como lo decretó la autoridad sanitaria federal, se cerraron todos los negocios no esenciales; pero, además, porque se enviaron a casa a cerca de 2300 trabajadores del municipio - de los 5000 que laboran-, a adultos mayores y a mujeres embarazadas. Esto les ha permitido no tener ningún caso de contagio en el gobierno municipal. Las plazas de Morelia se cerraron apenas hace ocho días.

El alcalde, Raúl Morón Orozco, considera que:

Se han tenido pocas incidencias de covid-19, porque la gente de Morelia ha sido muy disciplinada y porque se emprendieron tres acciones para contribuir a cuidar la salud de morelianas y morelianos. En primer lugar, en el cabildo se constituyó una "bolsa" de recursos financieros para este propósito; en segundo lugar, cuando se ordenó el cierre de todas las actividades no esenciales, se emprendió un programa alimentario para las personas que viven al día, diez mil paquetes alimentarios de 120 y 140 pesos, que ya se tenía, pero decidió ampliarse a 350 pesos. En total se distribuyeron a domicilio, 65 mil paquetes y convocamos a la solidaridad ciudadana, con lo que se creó un bono solidario, con el programa "Hacer Barrio Vale" con el que se entregaron 15 mil paquetes más. Estamos llegando a alrededor de 200 mil personas; además, la alcaldía decidió continuar con la obra pública, drenaje, agua potable, electricidad, y hemos reducido la deuda pública que teníamos de 500 millones de pesos; dejaremos al municipio sin deuda. La tercera acción frente a la pandemia será, no una "nueva normalidad", sino regresar a como estábamos trabajando antes de la pandemia, desde luego tenemos varios problemas, el presupuesto está como roto y (...) si llevamos agua a las comunidades (...), con los comerciantes, que están desesperados porque viven al día y ya quieren regresar; estamos dialogando para llegar a acuerdos. Les hemos dicho que nos faltan diez días. Antes teníamos diez casos, después ocho, siete y cada vez menos. Estoy convencido de que los gobiernos locales tienen que fortalecerse [para poder responder] porque somos la primera cara que ven los ciudadanos (Entrevista con alcalde de Morelia, 2020).

\section{Xalapa}

Xalapa tiene una población estimada de 513443 habitantes, para 2020; con 107 casos positivos acumulados y 28 casos positivos activos (al 8 de junio), con lo que se registra una tasa de 5.4 personas, por cada 100 mil habitantes, de casos positivos activos, la menor de los demás municipios y casi un tercio de la nacional. Una gran cantidad de su población también trabaja en el sector informal. Esto significa "que vive al día, en una situación laboral muy inestable, con una muy baja remuneración y que trabaja en la calle y requiere salir de su hogar" (Entrevista con alcade de Xalapa, 2020). 
El gobierno municipal de Xalapa, primero reorganizó el presupuesto para distribuir 7000 depensas en los hogares de alta y muy alta marginacion. Había colectivos que pedían apoyo, "pero dijimos no, vamos a ir directamente a los hogares para evitar la manipulación que somete al trabajador informal por líderes" (Entrevista con alcalde de Xalapa, 2020).

$\mathrm{El}$ otro propósito que se plantearon fue disminuir las reuniones públicas, como nos cuenta en entrevista, Pedro Hipólito Rodríguez Herrera, alcalde de Xalapa: "Una de las primeras decisiones fue cerrar bares y cantinas". Se trató de evitar que se reunieran los jóvenes colectivamente y en mayo "pusimos ley seca los fines de semana para reducir los conglomerados (...) y disminuyeron también los conflictos violentos y la violencia contra la mujer". Otra de las decisiones fue cerrar los espacios públicos: "Es que seguía llegando mucha gente a todos los parques de Xalapa". Los centros de educación superior, "que tienen mucha fuerza en Xalapa" suspendieron clases y la gente se fue a su comunidad, "Hasta el momento los Centros de Salud no han registrado ningún incremento notable. Nos propusimos que la curva fuera baja y lo hemos logrado, aunque entendemos que esto hará que se prolongue el periodo de contagio y vamos entonces a tener nuestra cúspide de contagio dentro de cuatro o cinco semanas".

En esta situación la alcaldía decidió aprovechar la coyuntura e impulsar algunos programas. "En la Comisión de Agua Potable teníamos mucha corrupción y teníamos escasez de agua, a pesar de que aquí llueve mucho,

ca

Las colonias populares

piden se apoye la economía

de los trabajadores por su cuenta,

de las MiPymes." hay mucho tandeo. Decidimos of recer un $50 \%$ de descuento en los estratos más bajos, un $25 \%$ en el medio y un $10 \%$ en el estrato alto" (Entrevista con alcalde de Xalapa, 2020). En cuanto a la alimentación, la alcaldía, a través del Consejo Municipal de Desarrollo Rural, impulsó un programa de gallinas ponedoras, huertos familiares eco-

lógicos y a las escuelas que capten el agua de lluvia. Para el tema de movilidad se impulsó un programa de ciclovías y el $80 \%$ de la obra pública se destinó a las colonias más pobres.

\section{Cuenavaca}

Cuernavaca fue el otro municipio estudiado, como parte de una investigación más amplia sobre la habitabilidad de las viviendas, frente al confinamiento dictado por la autoridad sanitaria nacional, para enfrentar la pandemia por covid-19. En esta ciudad no entrevistamos al alcalde, pero presentamos algunos resultados preliminares - de esta investigación en curso-, de 89 entrevistas, de las 200 que hemos realizado.

La gran mayoría de nuestros entrevistados de colonias populares (emergentes, tradicionales o rurales) habitan en viviendas de menos de 100 o $200 \mathrm{~m}^{2}$ y un tercio de los entrevistados, que viven en colonias populares emergentes, en viviendas de un solo cuarto (incluida la cocina), quizás los más afectados por el confinamiento; además, en el $20 \%$ de las viviendas de las colonias populares rurales, suelen vivir cinco o más personas. Más del $80 \%$ de las viviendas, de nuestros entrevistados de todas las colonias, son propias, aunque entre los que más rentan, se encuentra las personas de las colonias populares tradicionales y rurales.

En contraste con el problema de hacinamiento y espacio, que representa el hecho de que cinco personas o más habiten en viviendas con superficies menores de $100 \mathrm{~m}^{2}$, 
encontramos que, aproximadamente, uno de cada tres adultos mayores viven solos, con lo cual, surgen los siguientes cuestionamientos: ¿Cómo cumplir con las restricciones que en su cuidado les ha puesto la consigna federal de "Quédate en casa"? ¿Cómo proveerse de alimentos? A estas condiciones hay que agregar el severo problema del agua que se vive en Cuernavaca: una de cada dos viviendas — según nuestros entrevistados-, tiene problemas de acceso permanente al agua conectada a su vivienda y sólo la reciben algunos días de la semana. En 2016, el Sistema de Agua Potable y Alcantarillado de Cuernavaca recibió 59000 reclamos y se registraron 51.3 millones de litros de agua perdidos por cada kilómetro de la red de agua, indicador de los más altos del país (Sisniega Aspe y Salinas Ramírez, 2018).

Los problemas principales que señalan nuestros entrevistados, para cumplir con el llamado de "Quédate en casa", se jerarquizan de manera distinta dependiendo de dónde viven. Esto es importante porque en el $40 \%$ de los casos solo trabaja una persona en el hogar y en otro $40 \%$ trabajan dos personas. Por esto, quienes viven en las colonias populares emergentes y tradicionales, colocan en primer lugar la necesidad de ir a trabajar y de proveer alimentos. En las colonias populares emergentes, piden se apoye la economía de los trabajadores independientes y de las MiPymes; posponer pago de impuestos y no cobrar ni luz, ni agua; también los entrevistados, en general, proponen: solicitar a arrendadores diferimiento de pago de renta; la posposición de pagos hipotecarios a las instituciones financieras; habilitar espacios públicos para caminar y hacer deporte; habilitar la televisión pública y las herramientas de tecnologías de la información y comunicación, para poder cumplir con la "escuela en casa"; habilitar entregas a domicilio de alimentos y despensas, a los sectores más vulnerables, incluidos adultos mayores solos; y ser más estrictos con las personas que salen y organizan reuniones.

La mayoría de las personas entrevistadas de Cuernavaca estuvo de acuerdo con las acciones del gobierno federal ante la pandemia por Coronavirus, pero no con las que está realizando el gobierno estatal y las opiniones están divididas respecto a las acciones del

a

Uno de cada tres adultos mayores viven solos

¿Cómo cumplir 'Quédate en casa'...

cómo proveerse de alimientos?" gobierno municipal.

En Morelos la pandemia por Covid-19 tuvo pronto su cúspide a principios del mes de mayo; la tasa de casos positivos activos, por cada cien mil habitantes, lo ubicó en los primeros lugares nacionales, tercero, cuarto o quinto. Sin embargo, la comparación de la tasa de casos positivos activos de la primera semana de junio, con las de los 15 días anteriores, no indicaba crecimiento y denotaba hasta una ligera disminución. Cuernavaca, su capital, tenía 52 casos positivos activos al 8 de junio y tenía una tasa de 13 personas por cada 100 mil habitantes, casi el doble de la de Morelia y un punto menos que la nacional.

Ante la contingencia, la alcaldía de Cuernavaca ha realizado la mayoría de sus acciones mediante el Comité Municipal de Contingencia covid-19 (cMcc-19), que se constituyó con científicos del Instituto Nacional de Salud Pública, del Instituto de Biotecnología de la unam y la Universidad Autónoma del Estado de Morelos, todos establecidos en Cuernavaca. El CMCC-19 ha ido acompañando intensa y sistemáticamente las respuestas del gobierno municipal. Desde la tercera semana de marzo, en atención a las normas señaladas por la autoridad sanitaria nacional ante la contingencia, la alcaldía de Cuernavaca y el cmcc-19, a través del boletín 676, dictaron las siguientes disposiciones: 
- Cierre de gimnasios, escuelas, centros deportivos, guarderías, centros de desarrollo comunitario, Lienzo Charro, plazas de toros y parques.

- Cierre de balnearios, baños públicos (...) casinos, cines (...) jardines de eventos (...) centros nocturnos (...) cantinas, billares y centros de culto.

- Podrán funcionar las 24 horas agencias de inhumación, expendios de gas y gasolina, hoteles, sanatorios... hospitales... farmacias y tiendas de autoservicio... a partir de las 23:00 hasta las 7:00 horas del día siguiente.

- Las tiendas de conveniencia funcionarán de 8:00 a 23:00 horas con restricción de acceso.

- Funcionarán desde 7:00 hasta 23:00 horas expendios de pan, huevo, leche y tortilla, así como laboratorios de análisis clínicos.

- Los restaurantes (...) y cafeterías podrán operar desde 6:00 hasta 21:00 horas, [con una] distancia mínima de 1.5 metros entre cada mesa (...)

- Se restringirá visitas en asilos (...) estableciendo filtros sanitarios.

Conforme el número de casos fue aumentando, se fueron tomando más medidas por parte de la alcaldía de Cuernavaca; una de las más importantes fue el seguimiento de la concentración de casos por colonias: Centro, Antonio Barona, Altavista, Lomas de Ahuatlán y Santa María Ahuacatitlán, además de que, según la Alcaldía, "las ocupaciones con mayor número de casos reportados son empleados en general y personas dedicadas al hogar" (Comité Municipal de Contingencia covid-19, 2020, boletín 877 y 863). En marzo "se triplicó el número de casos sospechosos" (Comité Municipal de Contingencia covid-19, 2020, boletín 681 y 691) y se realizó "perifoneo, patrullajes y recorridos de inspectores para verificar cierre de locales comerciales y centros públicos (...) aplicando revisión y amonestaciones, según el caso. Además, el alcalde y empresarios del sector salud y educativo, discutieron estrategias para reactivar la economía, tras el paso del covid-19" (Comité Municipal de Contingencia covid-19, 2020, boletín 702). En estas sesiones entre alcaldía y los empresarios, "los integrantes del comité reiteraron la importancia de que los manejadores de alimentos refuercen las medidas sanitarias, al preparar los productos para la venta, como: usar cubrebocas, redes para el cabello, guantes, lavarse las manos (...) y limpiar superficies varias veces al día. (Comité Municipal de Contingencia covid-19, 2020, boletín 681). Se acordó con el Mercado Adolfo López Mateos que los giros no esenciales operarán conforme al avance del semáforo respectivo; también, el uso obligatorio del cubrebocas en el transporte público; y se consideró implantar la ley seca en Cuernavaca, pero no se hizo (Comité Municipal de Contingencia covid-19, 2020, boletín 812). Cuando sea el momento se activarán protocolos de "reapertura segura para giros comerciales y el plan de reactivación" (Comité Municipal de Contingencia covid-19, 2020, boletín 855), pero... después de siete días en la nueva normalidad... Cuernavaca mantiene el semáforo rojo del 8 al 14 de junio por lo que se pide utilizar cubrebocas y mantener la sana distancia" (Comité Municipal de Contingencia covid-19, 2020, boletín 877 y 863). Después de esto, se establecieron parámetros de cierre de todos los giros no esenciales, mientras el semáforo continúe en rojo; con reportes de entre 11 y 20 casos a la semana, durante 14 días, se pasará a la fase de semáforo naranja; después a la de color amarillo, con 10 casos positivos por dos semanas consecutivas, donde se abrirán otros lugares públicos con ciertas restricciones; y cuando se pase a semáforo verde, con cinco casos positivos o menos a la semana y durante 14 días consecutivos, para dar apertura a otros lugares públicos (Comité Municipal de Contingencia covid-19, 2020, boletín 855). 


\section{Conclusión}

Los gobienos municipales no tienen la autoridad sanitaria, para tomar medidas frente a la emergencia de salud que vivimos, ocasionada por el covid-19. Estas son facultades constitucionales, en primera instancia, del Gobierno de la República, encabezado por el poder ejecutivo federal, y en segunda instancia, de los gobiernos estatales. Sin embargo, siendo los municipios el tercer orden de gobierno constitucional, se ven exigidos por sus ciudadanos y obligados a tomar algunas medidas y acciones frente a la gravedad de la contingencia de salud, por ser la instancia de gobierno más cercana a los ciudadanos. Algunas de esas acciones, además, parecen tener importantes repercusiones en la contención de la contingencia.

Hemos presentado cuáles son las acciones y las medidas que han tomado algunos gobiernos municipales, como el abastecimiento del agua potable, que generalmente en todo el país está bajo la responsabilidad de sistemas operadores locales; el establecimiento de filtros/retenes sanitarios a la entrada del municipio o apoyos económicos a los gupos socioalmente más vulnerables y en mayor riesgo. Existen, además, otras medidas que los gobiernos municipales pueden tomar en cada uno de los 2457 municipios del país, como negociar con tianguistas u ofrecer apoyos a los grupos más vulnerables y los trabajadores del sector informal, al parecer los más expuestos, al no poder dejar de trabajar "porque viven al día". Esas medidas han sido tomadas por algunos gobiernos municipales, otros no las han implementado, argumentado problemas presupuestales. Sin embargo, estas medidas parece que tienen importantes consecuencias sobre la contingencia, por eso es importante ver qué gobiernos locales las asumen, para no simplemente reproducir y vigilar la diposición sanitaria nacional.

\section{Notas}

1 Los objetivos del programa son "contribuir al cumplimiento efectivo del derecho social a la alimentación y abatimiento de la desnutrición para el óptimo desarrollo humano de las personas que se encuentran por debajo de la línea de bienestar, mediante la dotación de leche con alto valor nutricional"

\section{Referencias}

Comité Municipal de Contingencia covid-19. (2020). Boletín 877 CMCC-19.

Ayuntamiento de Cuernavaca. http://cuernavaca.gob.mx/coronavirus/?page_ id $=774$

Comité Municipal de Contingencia covid-19. (2020). Boletín 863 CMCC-19. Ayuntamiento de Cuernavaca. http://cuernavaca.gob.mx/coronavirus/?page_ id $=774$

Comité Municipal de Contingencia covid-19. (2020). Boletin 855 CMCC-19.

Ayuntamiento de Cuernavaca. http://cuernavaca.gob.mx/coronavirus/?page_ id $=774$

Comité Municipal de Contingencia covid-19. (2020). Boletín 812 CMCC-19.

Ayuntamiento de Cuernavaca. http://cuernavaca.gob.mx/coronavirus/?page_ id $=774$ 
Comité Municipal de Contingencia covid-19. (2020). Boletín 702 CMCC-19.

Ayuntamiento de Cuernavaca. http://cuernavaca.gob.mx/coronavirus/?page_ id $=774$

Comité Municipal de Contingencia covid-19. (2020). Boletín 691 CMCC-19.

Ayuntamiento de Cuernavaca. http://cuernavaca.gob.mx/coronavirus/?page_ id $=774$

Comité Municipal de Contingencia covid-19. (2020). Boletín 681 CMCC-19.

Ayuntamiento de Cuernavaca. http://cuernavaca.gob.mx/coronavirus/?page_ id $=774$

Comité Municipal de Contingencia covid-19. (2020). Boletín 676 CMCC-19.

Ayuntamiento de Cuernavaca. http://cuernavaca.gob.mx/coronavirus/?page_ id $=774$

Centro de Investigaciones en Geografía Ambiental. (2020). COVID-19 Monitoreo de la Situación por Municipios. CIgA unam; Universidad Nacional Autónoma de México. Consultado el 7 de junio 2020 en: https://covid19.ciga.unam.mx/ datasets/f0f10e692a814fd8aa8afc7f8575f5d2

Sisniega Aspe, V., y Salinas Ramírez, R. (2018). SAPAC, al borde del colapso. Plan ciudadano para salvarlo. Centro de Investigación Morelos Rinde Cuentas, A.C. https://www. morelosrindecuentas.org.mx/sapac/upload/sapac-estudio-2018.pdf

\section{Entrevistas:}

Entrevista con Pedro Hipólito Rodríguez Herrero, alcalde de Xalapa, Veracruz, mayo 2020. Reunión convocada por la Red de Investigadores en Gobiernos Locales Mexicanos.

Entrevista con Clara Marina Brugada Molina alcaldesa de Iztapalapa, Ciudad de México, mayo 2020. Reunión convocada por la Red de Investigadores en Gobiernos Locales Mexicanos.

Entrevista con Raúl Morón Orozco, alcalde de Morelia, Michoacán, mayo 2020. Reunión convocada por la Red de Investigadores en Gobiernos Locales Mexicanos.

Para citar esta nota: Tapia, M. (3 de julio de 2020). Respuestas de los gobiernos locales a la pandemia por covid-19. Notas de coyuntura del CRIM No. 42, México, crim-unAm, 9 pp. 